\title{
Migration and Development
}

Herausgegeben von

Jürgen Meckl

Mit Beiträgen von

Bakewell, Oliver, Oxford, UK

Bauer, Thomas K., Essen

Czaika, Mathias, Freiburg

Docquier, Frédéric,

Louvain-La-Neuve, Belgium

Egger, Hartmut, Bayreuth

Felbermayr, Gabriel, Stuttgart

Lindley, Anna, London, UK

Rapoport, Hillel, Ramat Gan, Israel

Sinning, Mathias G., Canberra, Australia

Ziesemer, Thomas H.W., Maastricht, The Netherlands

Lucius \& Lucius - Stuttgart 2009 
Anschrift des Herausgebers des Themenheftes

Prof. Dr. Jürgen Meckl

Fachbereich Wirtschaftswissenschaften

Justus-Liebig-Universität Gießen

Licher Strasse 66

35394 Giessen

E-Mail: juergen.meckl@wirtschaft.uni-giessen.de

Bibliografische Information der Deutschen Nationalbibliothek

Die Deutsche Nationalbibliothek verzeichnet diese Publikation in der Deutschen Nationalbibliografie; detaillierte bibliografische Daten sind im Internet über http://dnb.d-nb.de abrufbar

ISBN 978-3-8282-0497-3

(C) Lucius \& Lucius Verlagsgesellschaft mbH $\cdot$ Stuttgart $\cdot 2009$

Gerokstraße 51, D-79184 Stuttgart

Das Werk einschließlich aller seiner Teile ist urheberrechtlich geschützt. Jede Verwertung außerhalb der engen Grenzen des Urheberrechtsgesetzes ist ohne Zustimmung des Verlags unzulässig und strafbar. Das gilt insbesondere für Vervielfältigungen, Übersetzungen und Mikroverfilmungen sowie die Einspeicherung und Verarbeitung in elektronischen Systemen.

Satz: Mitterweger \& Partner Kommunikationsgesellschaft mbH, Plankstadt Druck und Bindung: Neumann Druck, Heidelberg

Printed in Germany 